\title{
Revisão Toxicológica e Tratamento da Intoxicação pelo Êxtase
}

\author{
Larissa M. Veloso de Souza* \\ Lissa Hoshi* \\ Louise F. Lima* \\ Mila M. Santiago* \\ Marcelo Pinheiro R. Alves* \\ Leonardo O. Mendonça* \\ Marcelo Peixoto* \\ Túlio C. A. Alves** \\ Milena Pereira Pondé***
}

\begin{abstract}
RESUMO
O êxtase, cujo princípio ativo é a substância 3,4-metilenodioximetanfetamina (MDMA), é uma droga que causa uma série de efeitos adversos ao organismo, que podem ser classificados de acordo com o tempo e dose utilizados, ou com o mecanismo de ação e o sistema afetado. Essa droga atua sobre o sistema nervoso central, aumentando a secreção de serotonina, dopamina e noradrenalina em neurônios pré-sinápticos, além de impedir a destruição desses neurotransmissores pela enzima monoaminoxidase (MAO). O aumento da síntese de dopamina promove ativação dos centros de prazer dependentes de dopamina no cérebro. Entre os efeitos tóxicos mais freqüentes da droga estão a dificuldade de concentração, a depressão, a insônia, os ataques de pânico, a psicose, a hipertermia e a rabdomiólise. Tais sintomas precisam ser tratados rapidamente. O presente artigo objetiva mostrar os principais efeitos tóxicos do êxtase e o seu tratamento, bem como alertar sobre os diversos efeitos indesejáveis advindos do seu uso crônico e agudo.
\end{abstract}

Unitermos: Êxtase, efeitos tóxicos, revisão de literatura.

\section{Introdução}

O êxtase (ecstasy) é uma droga que foi desenhada em laboratório, tendo como princípio ativo a substância 3,4-metilenodioximetanfetamina (MDMA). Foi sintetizado em $1914^{1}$ pelo laboratório Merck da Alemanha como um moderador de apetite, mas nunca foi comercializado ${ }^{1}$. Na década de 1970 e 1980, o MDMA foi preconizado como adjuvante na psicoterapia, porque agiria na percepção de memórias

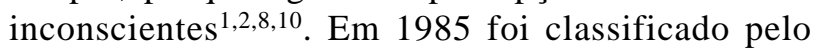
DEA (Drug Enforcement Administration) como droga nível 1 (alto potencial de abuso e sem uso médico atualmente aceito) e teve seu uso clínico proibido seguindo a orientação da OMS (Organização Mundial da Saúde) $)^{1,2,8,10}$. O êxtase é conhecido popularmente como: XTC, E, Adam, hug drug, M\&M, Beans, X, Clarity, Essence, Decadence $e^{1,2,11}$. O grau de pureza é variável em função da diversificação da mistura com outras drogas, tais como: LSD (ácido dietilamida lisérgico), anfetamina, efedrina, pseudo-efedrina, cetamina, cafeína, salicilatos, MDMA (3,4-metilenodioxi-N-etilanfetamina) ou o antitussígeno dextrometorfano (DTX $)^{4}$. Cada comprimido apresenta de $50 \mathrm{mg}$ a $150 \mathrm{mg}$ do princípio ativo e os custos para os consumidores variam de 10 a 50 dólares. Os principais usuários são adolescentes e adultos jovens, ricos ou de classe média, geralmente freqüentadores de raves, que são festas de longa duração, compreendendo a noite toda e parte da manhã, realizadas na clandestinidade, caracterizadas por música eletrônica e efeitos luminosos de laser, luzes negras, coloridas, estroboscópicas e globos de espelho. Os participantes ou ravers vestem-se, em geral, com roupas coloridas, usam adereços fluorescentes, valorizam moda e bodyart que incluem tatuagens e piercins. Os usuários são também adeptos da moda clubber e por isso detentores de maior poder aquisitivo, necessário para sustentar esse estilo de vida ${ }^{9}$. Essa moda é muito valorizada, menos colorida e psicodélica, mais geométrica e tecnológica. Tatuagens e piercins também fazem parte de cultura clubber.

\footnotetext{
* Acadêmico(a) da Escola Baiana de Medicina e Saúde Pública (EBMSP).

* Professor Titular de Farmacologia da EBMSP.

*** Professora Adjunta de Farmacologia da EBMS.
} 
A principal via de administração do êxtase é a oral, mas também existem as vias inalatória, retal e em forma de cigarros. As apresentações farmacêuticas de administração oral mais comuns são comprimidos, tabletes e cápsulas gelatinosas ou não. Na intoxicação pelo MDMA, os níveis plasmáticos atingem cerca de $7,72 \mathrm{mg} / \mathrm{l}^{11}$, com ampla distribuição tecidual e boa capacidade de atravessar a barreira hematoencefálica $^{11}$ e placentária ${ }^{12}$. Os efeitos surgem 30 a 60 minutos após o uso oral da droga, com pico em 90 minutos. Apresenta meia-vida de 7,6 horas. Seu metabolismo é hepático pela desaminação da cadeia lateral. A eliminação é predominantemente renal (65\%) e dependente de $\mathrm{pH}$; quanto mais ácido, mais facilmente a droga é eliminada ${ }^{11}$.

O objetivo deste estudo é realizar uma revisão da literatura médico-científica sobre os efeitos tóxicos por ocasião do uso do êxtase 3,4-metilenodioximetanfetamina (ecstasy), as suas eventuais conseqüências a curto e a longo prazo no organismo humano e o tratamento dessas complicações.

\section{Mecanismo de ação}

O 3,4-metilenodioximetanfetamina age sobre o sistema nervoso central (SNC) aumentando a secreção de serotonina, dopamina e noradrenalina em neurônios pré-sinápticos, sendo os neurônios serotoninérgicos os mais susceptíveis. O êxtase age também impedindo a recaptação desses neurotransmissores inibindo a ação da MAO (monoaminaoxidase). A liberação excessiva de serotonina e do ácido hidroxiindolacético (metabólito da serotonina) promove a auto-oxidação dessas substâncias, pois a MAO está inibida, formando metabólitos tóxicos que reagem com as enzimas e com as membranas neuronais causando neurodegeneração ${ }^{13}$. A longo prazo ocorre neurotoxicidade serotoninérgica pré e pós-sináptica caracterizada pela depleção de 5HT e 5HIAA, redução da enzima triptofano hidroxilase e redução da densidade de sítios de recaptação de $5 \mathrm{HT}^{11}$. Essa redução é seguida da depleção intraneural das reservas de $5 \mathrm{HT}$, que acaba por ativar receptores $2 \mathrm{~A} / 2 \mathrm{C}$ pós-sinápticos localizados em interneurônios GABA, resultando em redução desse neurotransmissor e da síntese de dopamina ${ }^{13}$.

$O$ efeito dopaminérgico do MDMA consiste na ativação dos centros de prazer dependentes de dopamina no cérebro ${ }^{17}$. Essa droga possui pouca atividade alucinógena relacionada ao estímulo de serotonina e estimula indiretamente a secreção de vasopressina por seus efeitos serotoninérgicos. O uso abusivo do MDMA está associado a acentuada hiponatremia e à síndrome de secreção inadequada de vasopressina. Pela estimulação dos neurônios serotoninérgicos há secreção de serotonina e as vias serotoninérgicas, da mesma forma, regulam a secreção do hormônio $\mathrm{ADH}^{7}$.

Há interação do êxtase com drogas inibidoras da MAO (monoaminoxidase) exacerbando os seus efeitos serotoninérgicos e simpatomiméticos ${ }^{2}$. O uso concomitante da maconha promove comprometimento cognitivo e de performance (estado de alerta, inteligência, atenção e memória) ${ }^{2,3,6,9,10,11}$. Efeitos subagudos no término da ação do MDMA são atenuados pela maconha, substância capaz de causar mudanças no ciclo de humor. Há relatos de início de esquizofrenia com o uso diário das duas drogas ${ }^{17}$. O uso do êxtase associado a álcool e Cannabis sativa diariamente provoca síndrome de despersonalização persistente. A associação com a cetamina pode causar problemas mentais graves ${ }^{17}$. Há indícios de que o uso concomitante de álcool e MDMA deprima o sistema imunológico ${ }^{9}$.

\section{Toxicologia}

Entre os efeitos buscados pelos usuários de êxtase estão a maior empatia, percepção de memórias inconscientes, sentimento de proximidade com as outras pessoas, aumento das sensações emotivas e sensuais, euforia, elevação da auto-estima e alteração da percepção visual ${ }^{1,9,11}$. Essa droga tem elevado potencial tóxico e pode conduzir a seqüelas pelo seu efeito cumulativo ${ }^{13}$.

O uso do êxtase causa vários efeitos que podem ser classificados de acordo com o tempo de uso, com a dose, com o mecanismo de ação e com o sistema afetado. Utilizando-se como parâmetros o tempo e a dose, as doses baixas promovem, como efeitos agudos, taquicardia, hipertensão, diminuição do apetite, tremor, trismo, bruxismo, náusea, insônia, cefaléia e sudorese ${ }^{2,9,11}$. Doses repetidas ou elevadas causam vômito, ataxia, nistagmo, aumento da acuidade para cores, alucinação visual, aumento da sensibilidade ao frio, dormência, formigamento nas extremidades e hepatite tóxica ${ }^{11}$. Nas reações de superdosagem podem ocorrer arritmias cardíacas, taquicardia, palpitação, hipertensão arterial, hipertermia e até morte ${ }^{11}$. Podem ocorrer efeitos residuais de ressaca no dia seguinte ao uso, que incluem insônia, fadiga, tontura e dores musculares, com efeitos persistentes por até duas semanas, como exaustão, fadiga, depressão, náusea, flashback e 
torpor $^{11}$. Esses efeitos devem-se à neurotoxicidade serotoninérgica da droga.

Durante a intoxicação aguda, alguns sintomas são decorrentes da estimulação simpática, ou seja, da intensa liberação de noradrenalina nos terminais présinápticos, o que pode causar diaforese, midríase, perturbação psicomotora e também alterações no aparelho cardiovascular ${ }^{1,2,9,11}$. As complicações que acometem o coração e o sistema de condução do sangue incluem hipertensão arterial sistêmica, arritmias, palpitações, bloqueio atrioventricular, choque cardiogênico e espasmo vasoconstritor difuso, ocasionando infarto intestinal ${ }^{1}$. O aumento da freqüência cardíaca ocorre devido ao aumento da atividade simpaticomimética e da permeabilidade iônica da membrana celular, resultando em aceleração do processo de auto-excitação no nó sinusal.

Uma das conseqüências imediatas do consumo de êxtase mais conhecida é a hipertermia, a qual contribui para outros efeitos sistêmicos graves, como a insuficiência renal aguda, a insuficiência hepática aguda, podendo ainda estimular a coagulação intravascular disseminada (CID), complicada por sangramento ${ }^{1,2,8,9,11}$.

A hipertermia deve-se à estimulação dos receptores alfa e beta-adrenérgicos, além da ativação do sistema simpático-adrenal. Em consequiência, observa-se a desnaturação protéica das fibras musculares esqueléticas (rabdomiólise). Assim como as demais drogas inibidoras da MAO, o êxtase pode causar necrose hepática maciça. Nos rins, observa-se acúmulo de proteínas nos capilares glomerulares e interstício renal, levando à glomerulonefrite e à necrose tubular, gerando insuficiência renal aguda. A CID é desencadeada por vasculites. $\mathrm{O}$ metabolismo natural do organismo também é afetado pelo êxtase e culmina em alterações, tais como: acidose, hipercalemia, hiponatremia e secreção intensa de hormônio antidiurético.

O sistema nervoso central é o principal alvo tanto dos efeitos desejáveis do êxtase quanto de sua ação tóxica pela estimulação serotoninérgica, levando a efeitos psicodélicos, como alucinações visuais e distorções cognitivas ${ }^{1,11}$. Detectam-se também a ocorrência de delírios, percepção alterada do tempo, redução da capacidade de atenção e cefaléia ${ }^{1,9,11}$. Outras conseqüências psiquiátricas são disforia, confusão mental, crises de paranóia, comportamento obsessivo, ataques de pânico, depressão, anorexia ou bulimia ${ }^{1,2,9,10,11}$. Outras manifestações também estão presentes na intoxicação cerebral com a 3,4metilenodioximetanfetamina e são representadas por fadiga, hiperreflexia, ataxia, nistagmo, compro- metimento visual, insônia, irritabilidade, convulsões, acidente vascular cerebral e até parada respiratória ${ }^{1,8}$.

O MDMA provoca alterações na circulação cerebral, como formação de saliências, dificuldades de enchimento vascular, fragmentação de vasos e hemorragias. A anorexia é causada pela liberação de dopamina no hipotálamo lateral. As alterações degenerativas irreversíveis do sistema nervoso central, mais precisamente nas sinapses serotoninérgicas, são resultantes de altas doses consumidas (300 mg ou mais) durante longo período de tempo. Esse efeito cumulativo pode trazer manifestações crônicas de ansiedade, ataques de pânico, depressão, fenômenos de flashbacks, psicoses e comprometimento cognitivo e da memória ${ }^{1,2,3,6,9,10,11}$. A empatia é provocada pela liberação aumentada de serotonina. A degeneração neuronal, as doenças vasculares cerebrais e o infarto do mesencéfalo são responsáveis pela perda de memória.

\section{Tratamento da intoxicação}

Entre os efeitos adversos mais freqüentes do êxtase estão a dificuldade de concentração, a depressão, a insônia e os ataques de pânico. Os sintomas mais graves são a psicose, a hipertermia, a rabdomiólise, a falência renal e hepática, as arritmias cardíacas e a hipertensão arterial. Todos eles precisam ser tratados de forma rápida e adequada para restabelecer a saúde do paciente.

Os efeitos toxicológicos da droga são potencializados com a repetição do uso e o aumento das doses, além de serem observados efeitos residuais. Dessa forma, a primeira medida de tratamento e supressão dos efeitos indesejáveis é a retirada do fármaco. Seguindo-se a isso, deve-se manter uma boa ventilação e perfusão sangüínea, por desobstrução das vias aéreas e acesso venoso. Como não existe um antídoto ou inibidor farmacológico do êxtase, o tratamento para os efeitos tóxicos decorrentes do seu uso baseia-se no tratamento dos sinais e dos sintomas da intoxicação.

Detectando-se hipertermia, é indicada a retirada das roupas, hidratação, umedecer partes do corpo e permanecer em local resfriado com o auxílio de ar condicionado, objetivando a termorregulação. Devese monitorar o nível de eletrólitos no sangue e o funcionamento dos órgãos, além de monitoramento da temperatura, evitando hipotermia. Em situações de hipertermia aguda extrema, deve-se utilizar gelo e, quando necessário, benzodiazepínicos no combate à síndrome serotoninérgica caracterizada por 
agitação, hipertermia e rigidez muscular. Deve-se ter o cuidado com uso de neurolépticos, que podem potencializar a hipertermia por diminuir a função dopaminérgica ${ }^{1,9}$.

Em situação de hipertensão e taquicardia, podese fazer uso de benzodiazepínicos. Não havendo resultado, utiliza-se a associação do bloqueador alfaadrenérgico fentolamina com o potente vasodilatador nitroprussiato. Em casos de arritmia cardíaca é indicado o uso do propranolol ou do esmolol ${ }^{9}$. Na ocorrência de vasoespasmo arterial, pode-se utilizar os derivados do ergot ${ }^{9}$, tal como a ergotamina, que provoca bloqueio alfa-adrenérgico.

Nos casos de hiponatremia associada a edema cerebral e sudorese, indica-se a administração de solução fisiológica de $\mathrm{NaCl}$ a $0,9 \%$ por via intravenosa ${ }^{1}$. Em quadros convulsivos, são usados benzoadiazepínicos. Em pacientes com risco de rabdomiólise, faz-se alcalinização da urina por meio de ampolas de bicarbonato de sódio e suplemento de potássio, podendo-se acrescentar manitol ${ }^{1}$.

\section{Discussão}

Nos estudos revisados não existe a confirmação de que a droga tomada fosse, de fato, o 3,4-metilenodioximetanfetamina, pois poucos foram os estudos em que se realizaram testes em pílulas ou em urina para verificar a presença de MDMA na forma farmacêutica ou no organismo. Além disso, o uso conjunto com outras drogas pode mascarar ou dar falsa impressão sobre os seus efeitos tóxicos. Por esses motivos, chama-se atenção para métodos de controle mais rigorosos nos estudos para que se possa determinar com um máximo de exatidão os efeitos adversos da droga.

Esta revisão toxicológica visa alertar a comunidade científica e a população em geral para os diversos efeitos indesejáveis advindos com o uso agudo ou crônico do êxtase. É de primordial importância a detenção dessas informações, haja vista que o uso da droga tem se disseminado, principalmente na população jovem. O tratamento eficaz da intoxicação por êxtase exige o pronto diagnóstico, o conhecimento prévio dos efeitos tóxico e das medidas terapêuticas. O ideal é que informações sobre a toxicologia do êxtase sejam difundidas não somente entre médicos, auxiliares e afins, mas também para toda a população, por campanhas preventivas, com difusão das informações por meio dos veículos de comunicação. A proposta é a conscientização do potencial tóxico do êxtase e dos seus malefícios à saúde, ressaltando a possibilidade de efeito letal.

\section{SUMMARY}

\section{Toxicological review and treatment of ecstasy} toxicity

Ecstasy is a drug that has 3,4-methylenedioxymethamphetamine or MDMA as its main active substance, and causes a variety of adverse effects that can be classified according to the length and dose taken, or according to the mechanism of action and system affected. It acts upon the Central Nervous System (CNS), increasing serotonin, dopamine and noradrenaline secretion in presynaptic neurons, and it also blocks the degradation of these neurotransmitters by the monoamine oxydase enzyme. Increase of dopamine synthesis activates dopamine-depending pleasure centers in the brain. The drug's most frequent effects are: difficulty in concentration, depression, insomnia, panic attacks, psychosis, hyperthermia and rhabdomyolysis. These reactions must be treated immediately. The present article focuses, through the toxicologic review and treatment of adverse effects of ecstasy, beside alerting about the several undesirable effects that occurs with the chronic or acute use of ecstasy.

\section{Keyw ords}

Ecstasy, toxic effects, bibliographic review.

\section{Referências}

1. Graeme KA. Pharmacologic advances in emergency medicine. New drugs of abuse - 3,4-methylenedioxymethamphetamine. Emergency Medicine Clinics of North America, 18(4), 2000. Disponível em: http:// www.mdconsult.com. Acesso em: 20 de abril de 2001.

2. Willians \& Wilkins. "Ecstasy": 3,4-methylenedioxymethamphetamine. Ellenhorn's Medical Toxicology, 2 ed., 1997. Disponível em: http://www.mdconsult.com. Acesso em: 20 de abril de 2001.

3. Yates B. Ecstasy may cause irreversible brain functioning. Journal of Neurology, Neurosurgery and Psychiatry, 68:719-25, 2000. Disponível em: http://www.jama.com. Acesso em: 1ㅇ de abril de 2001.

4. Baggto $\mathrm{M}$ et al. Chemical analysis of Ecstasy Pills. Disponível em: http://www.jama.com. Acesso em: $11 \mathrm{de}$ abril de 2001.

5. Gore SM. Fatal uncertainty: death-rate from use of Ecstasy or Heroin. The Lancet, 354:9, 1999. Disponível em: http://www.thelancet.com. Acesso em 5 de abril de 2001.

6. Engstrom JW, Hauser SL. Cognitive performance and recreational use of "Ecstasy". JAMA, 2000. Disponível em: http://www.jama.com. Acesso em: 11 de abril de 2001.

7. Henry JA et al. Low Dose MDMA ("ecstasy") induces vasopressin secretion. The Lancet, 351, 1998. Disponível em: http://www.thelancet.com. Acesso em 5 de abril de 2001. 
8. Rochester JA, Kirchner JT. Ecstasy (3,4-Methylenedioxymethamphetamine): history, neurochemistry and toxicology. Journal of American Board of Family Practice, 12(2):137-142, 1999. Disponível em: http:// www.mdconsult.com. Acesso em: 20 de abril de 2001.

9. Almeida SP, Silva MTA. Histórico, efeitos e mecanismos de ação do êxtase (3,4 metilenodioximetanfetamina): revisão de literatura. Revista Panamericana de Salud Publica, 6 de março de 2000. Disponível em: http:// www.scielosp.com. Acesso em 10 de abril de 2001.

10. Boot BR et al. MDMA (Ecstasy) neurotoxicity: assessing and communicating the risks. The Lancet 355:20, 2000. Disponível em: http://www.thelancet.com. Acesso em 17 de abril de 2001.

11. Ferigolo $\mathrm{M}$ et al. "Êxtase": revisão farmacológica. Rev Saúde Pública, 32(5):487-95, 1998.

12. McElahatton PR. Congenital anomalies after prenatal ecstasy exposure. The Lancet, 354:23, 1999. Disponivel em: http://www.thelancet.com. Acesso em 22 de abril de 2001.

13. McCann UD et al. Positron emission tomographic evidence of toxic effect of MDMA (Ecstasy) on brain serotonin neurons in human beigns. The Lancet, 352:31, 1998. Disponível em: http://www.thelancet.com. Acesso em 22 de abril de 2001.
14. Mathias R. "Ecstasy" damages the brain and impairs memory in humans. NIDA. Disponível em: http:// www.nida.nih.gov/NIDA_NOTES/NNVol14 N4/ Ecstasy.html. Acesso em: $17 \overline{\text { de }}$ abril de 2001.

15. Kish SJ et al. "Striatal serotonin is depleted in brin of a human MDMA user". Disponível em: http:// www.mdconsult.com. Acesso em: 20 de abril de 2001.

16. MDMA (Ecstasy). NIDA Notes. Disponível em: http:// www.nida.nih.gov/NIDA_NOTES/Infofax/Ecstasy.html. Acesso em: 17 de abril de 2001.

17. Jansen KLR. Adverse psychological effects associated with the use of ecstasy (MDMA) and their treatment. In: Sauders N. Ecstasy Reconsidered. p. 112-128, 1997. Disponível em: http://www.ecstasy.org/info/karl.html. Acesso em 11 de abril de 2001.

18. IOWA. Substance abuse-MDMA (3,4-methylenedioxymethamphetamine). University of IOWA, 2001. Disponível em: http://www.uiowa.edu/ shs/ecstasy.html. Acesso em: 10 de abril de 2001.

\section{Endereço para correspondência:}

Escola Baiana de Medicina e Saúde Pública

Departamento de Farmacologia

Rua Frei Henrique, 8 - Nazaré

CEP 40050-420 - Salvador, BA 